\title{
Predicting species composition in the tropical forests of the future: how tree species vary in their susceptibility to defaunation
}

\author{
Peter Williams ${ }^{1}$ and Jedediah Brodie ${ }^{2}$ \\ ${ }^{1}$ University of Montana Missoula \\ ${ }^{2}$ University of British Columbia
}

August 4, 2020

\begin{abstract}
Overhunting is extirpating large animals across tropical forests, affecting tree populations and potentially global carbon cycling. Species reliant on large-bodied seed dispersers may be particularly negatively affected. But defaunation also affects seed predation, trampling of seedlings, and conspecific density dependence. Therefore, defaunation predictions must incorporate multiple plant-animal interactions in the context of the entire tree life cycle. Because we cannot conduct such analyses for every species, we assess whether we can predict species' responses to defaunation based on phenotypic and demographic traits. Using population models, Monte Carlo simulations, and syntheses of demographic data, we found that responses to defaunation were best explained by how hunting altered seed predation, particularly for small-seeded angiosperms. How tree species will respond to defaunation still cannot be precisely predicted, but ascertaining how seed predation varies across species and hunting scenarios could greatly enhance our understanding of changing species composition and carbon dynamics in defaunated forests.
\end{abstract}

\section{INTRODUCTION}

Large vertebrates are being extirpated across the tropics, which affects the myriad tree species that interact with these animals (Kurten 2013; Dirzo et al. 2014). Such defaunation can affect plants in many ways. When trees are left without seed dispersers, for example, they may suffer population declines (Brodie et al. 2009; Culot et al. 2017; Rogers et al. 2017). Because many trees dispersed by large vertebrates are themselves large or have dense wood, defaunation may even induce shifts in tree species composition that reduce the aboveground biomass of tropical forests, with implications for the global carbon cycle (Brodie \& Gibbs 2009; Bello et al. 2015; Osuri et al. 2016; Peres et al. 2016). Furthermore, if hunting removes large predators, granivore populations could increase, leading to reduced seed survival (Galetti et al. 2015; Rosin \& Poulsen 2016). However, many tropical trees experience very strong conspecific density dependence (Harms et al. 2000; Peters 2003; Comita et al. 2010; Terborgh 2012), which implies that lower survival at early stages (e.g. through reduced seed dispersal or enhanced seed predation) could potentially be offset at the population level by ameliorated density dependence. Moreover, many of the hunted vertebrates are potent seed predators (Roldán \& Simonetti 2001; Donattiet al. 2009) or trample seedlings (Rosin et al. 2017), so removing these animals could benefit regeneration in certain plant species. It is critical, therefore, to assess how defaunation affects not just seed dispersal or seedling survival, but the entire life cycle of tropical trees.

Most previous studies on how defaunation affects trees (particularly those focusing on forest carbon impacts) have focused almost exclusively on reduced seed dispersal. These studies often simulate community composition in defaunated forests by 'removing' tree species that are large vertebrate-dispersed (Peres et al. 2016; Chanthorn et al. 2019) or that have large seeds (Bello et al. 2015; Osuriet al. 2016), and show that this can result in substantial reductions in aboveground biomass (i.e. carbon storage). Looking at empirical evidence from defaunated forests, though, the patterns are less clear. Populations of a tree species that significantly contributed to carbon stocks were indeed declining in defaunated forests in the Brazilian Atlantic 
Forest (Culot et al. 2017), but hunting-induced dispersal limitation appeared to have no impact on total tree biomass in Malaysian Borneo (Harrison et al. 2013).

The best way to predict the effects of defaunation on tree species is to conduct population-level, whole-lifecycle analyses. But such analyses are very resource-intensive, precluding the evaluation of all tropical tree species over any conservation-relevant time frame. Therefore, it is important to try to ascertain whether we can predict a prioriwhich tree species might be susceptible to defaunation, for example based on their phenotypic traits. Defaunation responses could potentially vary with morphological traits such as seed size, which may affect seed predation (Mendoza \& Dirzo 2007), or with ecological traits such as dispersal mode, which could affect susceptibility to disperser loss (Peres et al. 2016). However, given the multiple effects of defaunation on plants at different life stages, what matters is how all of the impacts combine to influence overall population dynamics (Harrison et al. 2013) and whether this varies with life history characteristics. Demographic rates for tropical trees tend to be correlated with physical traits (Poorter et al. 2008) that are easier to measure and collect. If we could use combinations of physical traits to ascertain a given tree species' susceptibility to defaunation-induced population decline, we could better predict the changing species composition of defaunated tropical forests.

Here we synthesized data on tropical tree populations, the multiple impacts of defaunation across the plant life cycle, and tree morphological and demographic traits to assess whether we can predict how trees with different traits vary in their responses to hunting-induced population declines. We used density-dependent demographic models and Monte Carlo simulations that incorporate data on all facets of tree life history, defaunation effects, and the (often substantial) uncertainty in these factors. Specifically, our objective was to determine whether any traits or trait combinations were associated with tree susceptibility to a range of different defaunation effects.

\section{METHODS}

\section{Population matrices}

We obtained tropical tree population matrices for 58 species from the COMPADRE Plant Matrix Database (Salguero-Gómez et al. 2015). Population matrices were included in the analysis if they (1) were classified as a 'tree' or 'palm', (2) were from tropical or subtropical regions, (3) did not include clonal reproduction, (4) included life stages that could be categorized as 'seedling', 'juvenile', and 'adult' stage classes, (5) included transitions between major stages, and (6) included populations in 'unmanipulated' or 'unmanaged' habitat treatments. For species with multiple population matrix studies that fit these criteria, we selected the study that included the most populations. For studies with multiple matrices of a single species $(\mathrm{N}=25)$, we averaged matrix elements across populations to create a composite representative matrix for that species. Averaging matrices across populations did not affect the ultimate results of our study (Table S1 in supporting information).

We reduced the sizes of each matrix to $4 \times 4$ with the following stages: seed, seedling, juvenile, and adult. If a matrix had multiple sub-classes within a single stage (e.g. multiple 'adult' size classes), we combined stage classes such that the new composite matrix had the same stable stage distribution and the same asymptotic growth rate as the original matrix (Yearsley \& Fletcher 2002). Some studies considered 'seedlings' to be plants with a diameter at breast height $<1 \mathrm{~cm}$, while in most other studies 'seedlings' were individuals under a certain height (though the exact threshold varied across species). For our 'seedling' stage, we followed the definitions used by each study. 'Juvenile' encompassed non-reproductive stages between seedling and adult. If matrices lacked a seed stage (i.e. the fecundity element in the matrix was the number of seedlings produced), we used seed survival values from the original studies to add a seed stage. For species that lacked seed survival data, we created a seed stage using the average seed survival value among non-cycads or cycads. We divided our data in this way because cycads had significantly higher seed survival than other major lineages (i.e. conifer, magnoliid, eudicot, monocot), but seed survival was not significantly different among other clades (Figure S1). Using averaged seed survival values did not affect the ultimate results of our study (Table S1). No regression transitions, such as juveniles becoming seedlings, were allowed. 


\section{Trait data}

We assigned dispersal mode (abiotic versus animal-dispersed) based on species- or genus-level data in the TRY database or, if such data were missing, based on searching the literature. We defined tree species as 'large vertebrate dispersed' if the list of their dispersers did not include any small-bodied rodents (families Muridae, Sciuridae, Heteromyidae, Cricetidae, or Echimyidae) or non-corvid passerine birds. Lists of dispersers were obtained from the TRY database if possible and from the literature if not (information sources for all species presented in Table S2).

Seed mass, wood density, specific leaf area, and adult plant height were gleaned from the TRY Plant Trait Database (Kattge et al. 2011) if available, or otherwise taken from the literature (Table S3). We chose these traits because they are some of the most commonly measured functional traits and ones that are often used in functional trait studies (e.g. Adler et al. 2014; Kunstler et al. 2016). When we could not find species-level data, we used median genus-level values for wood density (10 species; $17 \%$ of total) and SLA (17 species; $29 \%$ ). The TRY database did not include any wood density data for cycads, but we found stem density volume and mass estimates in the literature (Cousins et al. 2011).

\section{Defaunation model}

Using population matrices from the 64 tree species, we simulated the effects of defaunation by altering particular matrix elements. Specifically, we used the following parameters to estimate defaunation impacts: (1) the change in seed predation rate after defaunation $(p),(2)$ the proportion of seeds dispersed before $\left(d_{\text {base }}\right)$ and after $\left(d_{\text {defaun }}\right)$ defaunation, (3) the difference in survival between dispersed and undispersed seeds $(a)$, (4) the difference in survival between dispersed and undispersed seedlings $(b),(5)$ the strength of conspecific negative density dependence $(\beta)$, and (6) the change in trampling-induced seedling mortality after defaunation $(t)$. For $p$, we compiled a list of values based on published meta-analyses (Kurten 2013; Gardner et al.2019) and our own literature search (Table S4). For other parameters $\left(d_{\text {base }}, d_{\text {defaun }}, a, b\right.$, and $\beta$ ), we compiled lists of values for based on published meta-analyses (Kurten 2013; Comita et al. 2014; Lamannaet al. 2017). Because research that quantified the effects of reduced seedling trampling was limited, we compiled a list of values fort based on published studies (Roldán \& Simonetti 2001; Rosinet al. 2017). Table S5 provides a full list of model parameters and the sources of the data that we used to estimate them.

We ran 10,000 iterations of the model, randomly drawing values in each iteration for $p, d_{\text {base }}, d_{\text {defaun }}, a$, $b, \beta$, and $t$ from lists of parameter values (see Table S5). Within each iteration, we assembled a 'baseline' population matrix for each tree species from the chosen parameter values. To make the baseline population matrix, we used the original matrices for each species, but we divided both seeds and seedlings into dispersed versus undispersed stages (Figure 1). For each species, we used original vital rates and the parameters $d_{\text {base }}$ , $a$, and $b$, to calculate the numbers of seeds dispersed or undispersed, the baseline survival rates for dispersed and undispersed seeds, and the maximum survival rates for dispersed and undispersed seedlings (Equations S1-S8). Seedling survival can depend on the density of adult trees $(A)$ due to Janzen-Connell effects (Zhu et al. 2015); so we incorporated density dependence using a Ricker function (Ricker 1954):

$\varsigma_{\lambda}=a \epsilon^{\beta A}(1)$

where $s_{l}$ is the survival of seedlings (dispersed or undispersed), $a$ is the maximum survival of seedlings (dispersed or undispersed) in the absence of adult trees, and $\beta$ is the strength of conspecific negative densitydependence.

For each species in each iteration, we also created a 'defaunation' population matrix (Figure 1). The defaunation matrices had three key differences from the baseline matrices. First, the probability of seed dispersal for 'large vertebrate dispersed' tree species was changed to $d_{\text {defaun }}$. For other species, the probability of dispersal remained set to $d_{\text {base }}$. Second, we used $p$, the change in seed predator-induced mortality between baseline and defaunation scenarios, to alter the survival of dispersed and undispersed seeds (Equations S11-S12). Third, we used $t$, the change in trampling-induced seedling mortality between the baseline and defaunation scenarios, to alter the maximum survival (before accounting for density-dependence) of dispersed 
and undispersed seedlings (Equations S13-S15). With these new maximum survival values, actual seedling survival was calculated using the same density-dependent function (Equation 1).

Once the baseline and defaunation matrices of each species had been built, we simulated 120 years of population growth under baseline and defaunation scenarios. Each simulation began with an initial density of 25 adults $\mathrm{ha}^{-1}$, based on typical values assembled by LaManna et al. (Lamanna et al. 2017), while the rest of the initial population vector was calculated based on the proportions of adults to other life stages at stable stage distribution. We then calculated the 'defaunation response' as the final number of adults in the defaunation population divided by the final number of adults in the baseline population.

For each of the 10,000 interactions, we drew new values for our defaunation parameters $\left(p, d_{\text {base }}, d_{\text {defaun }}, a\right.$ $, b, \beta$, and $t$ ) to set a new defaunation scenario, simulated baseline and defaunation population dynamics for each species, and calculated defaunation response of each species for that scenario.

\section{Model output}

To analyse the output of the simulation model, we ran linear models of defaunation parameters and species traits (predictor variables) against 'defaunation response' (response variable; $\log _{10}$-transformed to account for extreme skew). We assessed a global model as well as models representing all subsets of predictor variables, and selected the model with the lowest BIC value as our top 'additive model' using the MuMIn package (Barton 2019) in R (R Core Team 2018). We used BIC rather than AIC because it more strongly penalizes complex models, and so is better for identifying a few variables with strong effect (Burnham \& Anderson 2004). We also created 'interaction models' to assess whether the inclusion of statistical interaction terms between main-effect variables in the additive model improved model fit.

For the additive model and each interaction model, we used therelaimpo package (Grömping 2006) in $\mathrm{R}$ to calculate the relative importance of each variable, measured as the contribution of each variable to the model's $\mathrm{R}^{2}$, following the Lindeman-Merenda-Gold (Lindeman et al. 1980) method.

\section{RESULTS}

Our defaunation model included data from 58 species from 53 genera in 30 families (Table S2). With 10,000 iterations per species, this created 58,000 'defaunation response' values across all defaunation scenarios.

In our additive model, the most important variable for explaining defaunation response was seed predation (model coefficient $\beta=-0.153$ ), explaining $6.82 \%$ of model variance, followed more distantly by seedling trampling $(\beta=-0.076,1.69 \%$ of variance; Figure 2$)$. Across all species, changes in seed predation predicted defaunation response (Figure 3A). No phenotypic traits were associated with defaunation response across species.

Important statistical interactions emerged between seed predation and (i) angiosperm versus gymnosperm $(\beta$ $=0.146)$, (ii) seed mass $(\beta=0.097)$, and (iii) specific leaf area $(\beta=-0.073$; Figure S2). When defaunation increased seed predation, populations of angiosperms (Figure 3B), small-seeded species (Figure 3C), and taxa with very high specific leaf area (Figure S3) declined. Gymnosperms, large-seeded species, and taxa with low to medium specific leaf area were relatively unaffected by defaunation regardless of seed predation.

Several phenotypic traits were significantly correlated with species vital rates (Table S6). Seed mass, in particular, was positively related to juvenile survival $(r=0.419, P=0.001)$ and adult survival $(r=0.333$, $P=0.011)$, but was negatively related to adult fecundity $(r=-0.354, P=0.006)$. Fecundity also correlated with whether a species was an angiosperm or a gymnosperm $(r=0.480, P<0.001)$ and was positively related to tree height $(r=0.339, P=0.009)$.

\section{DISCUSSION}

Overhunting can alter many plant-animal interactions, with consequences ranging from altered dynamics of tropical tree populations (Brodieet al. 2009; Culot et al. 2017) to potential declines in forest carbon storage (Bello et al. 2015; Peres et al.2016; Chanthorn et al. 2019). But we still have a limited understanding of 
how the multiple effects of defaunation combine to affect tree populations. Our results suggest that how a tropical tree species responds to defaunation is primarily based on whether and how the predation of its seeds is altered by hunting. But unfortunately, even knowledge about altered seed predation rates still confers only limited ability to predict population-level responses. Moreover, our knowledge about how defaunation affects seed predation remains limited. Granivory could increase (Galetti et al. 2015; Rosin \& Poulsen 2016; Culot et al. 2017) or decrease (Wright et al. 2000; Roldán \& Simonetti 2001; Beckman \& Muller-Landau 2007) under defaunation and, even in the same forest, species can differ wildly in how seed predation rates change in response to defaunation (Guariguataet al. 2000; Rosin \& Poulsen 2016). In our model, we applied changes in seed predation equally to all of our species, but our results might have changed if seed predation varied according to species traits. For example, Mendoza and Dirzo (2007) hypothesized that smaller seeds, whose small-bodied predators would likely remain extant even in highly defaunated systems, would face higher predation pressure than larger seeds, whose predators would be eliminated by overhunting. However, we conducted a review of studies that measured seed predation in defaunation versus non-defaunation conditions (Table S4) and found that seed size did not predict changes in predation pressure (Figure S4). Though seed size did not correlate with change in seed predation, our results show that small-seeded angiosperms were more demographically sensitive to changes in seed predation than gymnosperms or large-seeded species. This result provides a potential focus for future research: understanding exactly how defaunation affects seed predation may be particularly important for assessing the vulnerability of small-seeded angiosperms.

Defaunation can also affect later life-stages via changes in herbivory or other physical damage (Gardner et al. 2019). We did not include such effects in our model because of very scant data connecting defaunation to changes in sapling or adult vital rates. Removing large-bodied ecosystem engineers such as elephants may impact older plant life stages (Poulsen et al. 2018), though the distribution of such megafauna and their foraging behaviour are highly variable across the world's tropical forests. While changes in herbivores and ecosystem engineer abundances may significantly impact local plant communities (Luskin et al. 2017), it is difficult to consider these factors in a global assessment of defaunation.

Surprisingly, factors such as dispersal mode and the strength of density dependence were unrelated to demographic responses to defaunation in our model. Previous studies have assumed that dispersal limitation was the most important effect of defaunation (Terborgh 2013), leading to predictions that defaunation-induced losses of seed dispersal will reduce carbon storage (Brodie \& Gibbs 2009; Bello et al. 2015; Dantas de Paula et al. 2018; Chanthorn et al. 2019). However, given that defaunation alters seed predation as well as seed dispersal, and that altered seed dispersal was unrelated to population-level defaunation impacts, overhunting may not necessarily cause the widespread replacement of heavy-wooded, vertebrate-dispersed species by lighterwooded species (with other means of dispersal) that have been predicted. Therefore, whether defaunation will result in major losses of forest carbon remains unclear. Indeed, not all defaunated forests show decreases in biomass (Harrison et al. 2013; Bagchiet al. 2018). Despite attempts to model the impacts of defaunation on tropical tree communities and ecosystems via altered seed dispersal alone, our study shows that there is too much uncertainty surrounding other defaunation effects - notably seed predation - to currently be able to make accurate predictions about cascading impacts on populations and ecosystem processes.

Likewise, phenotypic traits conferred little ability to predict defaunation responses across species. While many of the traits did correlate with the vital rates that determine population dynamics, these correlations were relatively weak (Table S6). Importantly, of the traits that we assessed, only seed mass significantly correlated with adult survival, which, in long-lived woody plants, influences population dynamics more than any other vital rate (Silvertown et al. 1993; Zuidema 2000). Because large seeds are unlikely to be dispersed by small vertebrates, many studies have assumed that large-seeded species are the most vulnerable to defaunation (e.g. Bello et al. 2015; Osuriet al. 2016). However, in our study, large-seeded species were less responsive to defaunation because of their lower adult mortality. Our smaller-seeded species had stronger responses to defaunation, but the direction of the response was mediated by how defaunation affected seed predation. As seed mass does not predict changes in seed predation (Figure S4), we still cannot use this trait to assess $a$ priorispecies' responses to defaunation. 
What, then, can we predict about the consequences of defaunation for tropical trees? We know from studies of individual species that defaunation can cause significant population declines (Wright \& Duber 2001; Culot et al. 2017), but we still have limited ability to scale up these observations to predict patterns at the community or ecosystem level. Phenotypic traits and even demographic vital rates are not sufficient to predict defaunation effects. However, our results highlight a fruitful direction for future research; an improved understanding of seed predation - how it affects tree populations and how it is affected by defaunation — would greatly reduce uncertainty around the cascading impacts of defaunation. This will improve the accuracy of predictions of the global consequences of overhunting for tropical forests.

\section{Acknowledgements}

This work was supported by the University of Montana. We thank W. Lowe, A. Luis, J. Maron, K. McConkey, and J. Millspaugh for discussions about these topics and advice on the analysis.

\section{REFERENCES}

Adler, P.B., Salguero-Gómez, R., Compagnoni, A., Hsu, J.S., Ray-mukherjee, J., Mbeau-Ache, C., et al. (2014). Functional traits explain variation in plant life history strategies. PNAS , 111, 740-745.

Bagchi, R., Swamy, V., Latorre Farfan, J.P., Terborgh, J., Vela, C.I.A., Pitman, N.C.A., et al. (2018). Defaunation increases the spatial clustering of lowland Western Amazonian tree communities. J. Ecol., 106, 1470-1482.

Barton, K. (2019). MuMIn: Multi-Model Inference.

Beckman, N.G. \& Muller-Landau, H.C. (2007). Differential Effects of Hunting on Pre-Dispersal Seed Predation and Primary and Secondary Seed Removal of Two Neotropical Tree Species. Biotropica , 39, 328-339.

Bello, C., Galetti, M., Pizo, M.A., Magnago, L.F.S., Rocha, M.F., Lima, R.A.F., et al. (2015). Defaunation affects carbon storage in tropical forests. Sci. Adv. , 1, 1-10.

Brodie, J.F. \& Gibbs, H.K. (2009). Bushmeat hunting as climate threat.Science (80-. ). , 326, 364-365.

Brodie, J.F., Helmy, O.E., Brockelman, W.Y. \& Maron, J.L. (2009). Bushmeat poaching reduces the seed dispersal and population growth rate of a mammal-dispersed tree. Ecol. Appl. , 19, 854-863.

Burnham, K.P. \& Anderson, D.R. (2004). Multimodel Inference Understanding AIC and BIC in Model Selection. Sociol. Methods Res., 33, 261-304.

Chanthorn, W., Hartig, F., Brockelman, W.Y., Srisang, W., Nathalang, A. \& Santon, J. (2019). Defaunation of large-bodied frugivores reduces carbon storage in a tropical forest of Southeast Asia. Sci. Rep. , 9, 1-9.

Comita, L.S., Muller-Landau, H.C., Aguilar, S. \& Hubbell, S.P. (2010). Asymmetric Density Dependence Shapes Species Abundances in a Tropical Tree Community. Science (80-. ). , 329, 330-332.

Comita, L.S., Queenborough, S.A., Murphy, S.J., Eck, J.L., Xu, K., Krishnadas, M., et al. (2014). Testing predictions of the Janzen-Connell hypothesis: A meta-analysis of experimental evidence for distance- and density-dependent seed and seedling survival. J. Ecol. , 102, 845-856.

Cousins, S.R., Williams, V.L. \& Witkowski, E.T.F. (2011). Quantifying the Trade in Cycads (Encephalartos Species) in the Traditional Medicine Markets of Johannesburg and Durban, South Africa. Econ. Bot. , 65, $356-370$.

Culot, L., Bello, C., Batista, J.L.F., do Couto, H.T.Z. \& Galetti, M. (2017). Synergistic effects of seed disperser and predator loss on recruitment success and long-term consequences for carbon stocks in tropical rainforests. Sci. Rep. , 7, 1-8.

Dantas de Paula, M., Groeneveld, J., Fischer, R., Taubert, F., Martins, V.F. \& Huth, A. (2018). Defaunation impacts on seed survival and its effect on the biomass of future tropical forests. Oikos , 127, 1526-1538. 
Dirzo, R., Young, H.S., Galetti, M., Ceballos, G., Isaac, N.J.B. \& Collen, B. (2014). Defaunation in the Anthropocene. Science (80-. ). , 345, 401-406.

Donatti, C.I., Guimarães, P.R. \& Galetti, M. (2009). Seed dispersal and predation in the endemic Atlantic rainforest palm Astrocaryum aculeatissimum across a gradient of seed disperser abundance.Ecol. Res. , 24, $1187-1195$.

Galetti, M., Bovendorp, R.S. \& Guevara, R. (2015). Defaunation of large mammals leads to an increase in seed predation in the Atlantic forests. Glob. Ecol. Conserv. , 3, 824-830.

Gardner, C.J., Bicknell, J.E., Baldwin-Cantello, W., Struebig, M.J. \& Davies, Z.G. (2019). Quantifying the impacts of defaunation on natural forest regeneration in a global meta-analysis. Nat. Commun. , 10, 1-7.

Grömping, U. (2006). Relative Importance for Linear Regression in R: The Package relaimpo. J. Stat. Softw. , 17, 1-27.

Guariguata, M.R., Adame, J.J.R. \& Finegan, B. (2000). Seed Removal and Fate in Two Selectively Logged Lowland Forests with Constrasting Protection Levels. Conserv. Biol. , 14, 1046-1054.

Harms, K.E., Wright, S.J., Caldero, O., Hernandez, A. \& Herre, E.A. (2000). Pervasive density-dependent recruitment enhances seedling diversity in a tropical forest. Nature , 404, 493-495.

Harrison, R.D., Tan, S., Plotkin, J.B., Slik, F., Detto, M., Brenes, T., et al. (2013). Consequences of defaunation for a tropical tree community. Ecol. Lett. , 16, 687-694.

Kattge, J., Díaz, S., Lavorel, S., Prentice, I.C., Leadley, P., Bönisch, G., et al. (2011). TRY - a global database of plant traits. Glob. Chang. Biol. , 17, 2905-2935.

Kunstler, G., Falster, D.S., Coomes, D.A., Hui, F., Kooyman, R.K., Laughlin, D.C., et al. (2016). Plant functional traits have globally consistent effects on competition. Nature , 529, 204-207.

Kurten, E.L. (2013). Cascading effects of contemporaneous defaunation on tropical forest communities. Biol. Conserv. , 163, 22-32.

Lamanna, J.A., Mangan, S.A., Alonso, A., Bourg, N.A., Brockelman, W.Y., Bunyavejchewin, S., et al. (2017). Plant diversity increases with the strength of negative density dependence at the global scale.Science (80-. ). , 356, 1389-1392.

Lindeman, R.H., Merenda, P.F. \& Gold, R.Z. (1980). Introduction to Bivariate and Multivariate Analysis . Glenview IL.

Luskin, M.S., Brashares, J.S., Ickes, K., Sun, I., Fletcher, C., Wright, S.J., et al. (2017). Cross-boundary subsidy cascades from oil palm degrade distant tropical forests. Nat. Commun. , 8, 1-8.

Mendoza, E. \& Dirzo, R. (2007). Seed-size variation determines interspecific differential predation by mammals in a neotropical rain forest. Oikos , 116, 1841-1852.

Osuri, A.M., Ratnam, J., Varma, V., Alvarez-Loayza, P., Hurtado Astaiza, J., Bradford, M., et al. (2016). Contrasting effects of defaunation on aboveground carbon storage across the global tropics. Nat. Commun. , $7,11351$.

Peres, C.A., Emilio, T., Schietti, J., Desmoulière, S.J.M. \& Levi, T. (2016). Dispersal limitation induces long-term biomass collapse in overhunted Amazonian forests. Proc. Natl. Acad. Sci. , 113, 892-897.

Peters, H.A. (2003). Neighbour-regulated mortality: the influence of positive and negative density dependence on tree populations in species-rich tropical forests. Ecol. Lett. , 6, 757-765.

Poorter, L., Wright, S.J., Paz, H., Ackerly, D.D., Condit, R., Ibarra-Manriquez, G., et al. (2008). Are functional traits good predictors of demographic rates? Evidence from five neotropical forests.Ecology , 89, $1908-1920$. 
Poulsen, J.R., Rosin, C., Meier, A., Mills, E., Nunez, C.L., Koerner, S.E., et al. (2018). Ecological consequences of forest elephant declines for Afrotropical forests. Conserv. Biol. , 32, 559-567.

R Core Team. (2018). R: A language and environment for statistical computing.

Ricker, W.E. (1954). Stock and recruitment. J. Fish. Res. Board Canada, 11, 559-623.

Rogers, H.S., Buhle, E.R., Hille Ris Lambers, J., Fricke, E.C., Miller, R.H. \& Tewksbury, J.J. (2017). Effects of an invasive predator cascade to plants via mutualism disruption. Nat. Commun. , 8, 1-8.

Roldán, A.I. \& Simonetti, J.A. (2001). Plant-Mammal Interactions in Tropical Bolivian Forests with Different Hunting Pressures. Conserv. Biol. , 15, 617-623.

Rosin, C. \& Poulsen, J.R. (2016). Hunting-induced defaunation drives increased seed predation and decreased seedling establishment of commercially important tree species in an Afrotropical forest.For. Ecol. Manage. , 382, 206-213.

Rosin, C., Poulsen, J.R., Swamy, V. \& Granados, A. (2017). A pantropical assessment of vertebrate physical damage to forest seedlings and the effects of defaunation. Glob. Ecol. Conserv. , 11, 188-195.

Salguero-Gómez, R., Jones, O.R., Archer, C.R., Buckley, Y.M., Che-castaldo, J., Caswell, H., et al. (2015). The COMPADRE Plant Matrix Database: an open online repository for plant demography.J. Ecol. , 103, $202-218$.

Silvertown, J., Franco, M., Pisantyt, I. \& Mendoza, A. (1993). Comparative Plant Demography-Relative Importance of Life-Cycle Components to the Finite Rate of Increase in Woody and Herbaceous Perennials. J. Ecol. , 81, 465-476.

Terborgh, J. (2012). Enemies Maintain Hyperdiverse Tropical Forests.Am. Nat. , 179, 303-314.

Terborgh, J. (2013). Using Janzen-Connell to predict the consequences of defaunation and other disturbances of tropical forests. Biol. Conserv. , 163, 7-12.

Wright, S.J. \& Duber, H.C. (2001). Poachers and Forest Fragmentation Alter Seed Dispersal, Seed Survival, and Seedling Recruitment in the Palm Attalea butyraceae, with Implications for Tropical Tree Diversity.Biotropica , 33, 583-595.

Wright, S.J., Zeballos, H., Domínguez, I., Gallardo, M.M., Moreno, M.C. \& Ibáñez, R. (2000). Poachers Alter Mammal Abundance, Seed Dispersal, and Seed Predation in a Neotropical Forest. Conserv. Biol. , 14, $227-239$.

Yearsley, J.M. \& Fletcher, D. (2002). Equivalence relationships between stage-structured population models. Math. Biosci., 179, 131-143.

Zhu, Y., Comita, L.S., Hubbell, S.P. \& Ma, K. (2015). Conspecific and phylogenetic density-dependent survival differs across life stages in a tropical forest. J. Ecol. , 103, 957-966.

Zuidema, P.A. (2000). Demography of exploited tree species in the Bolivian Amazon. PROMAB. 


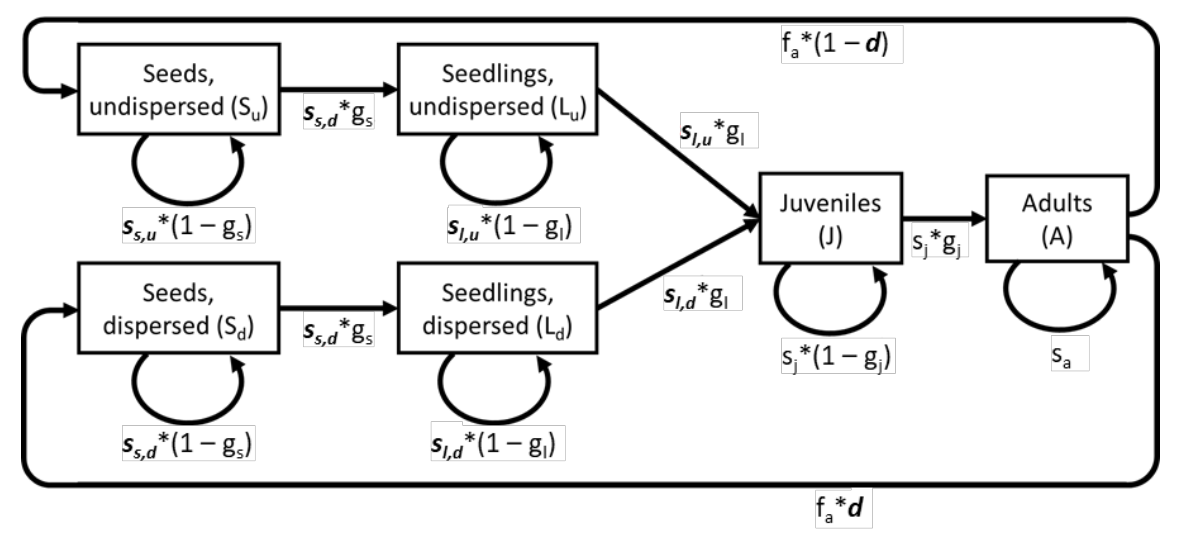

Figure 1.

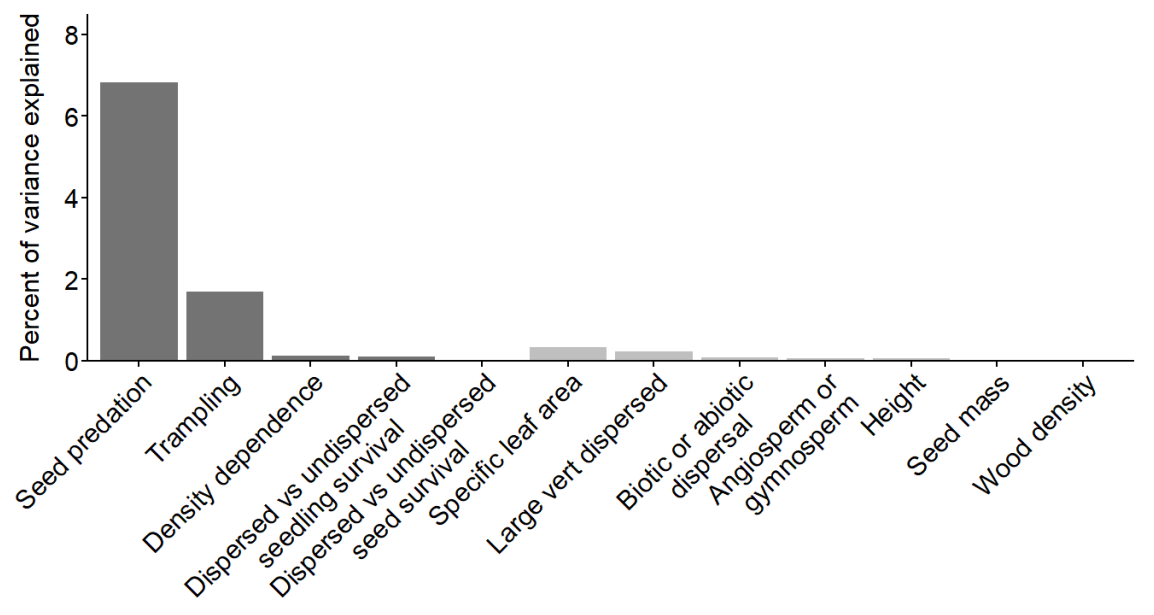

Figure 2. 


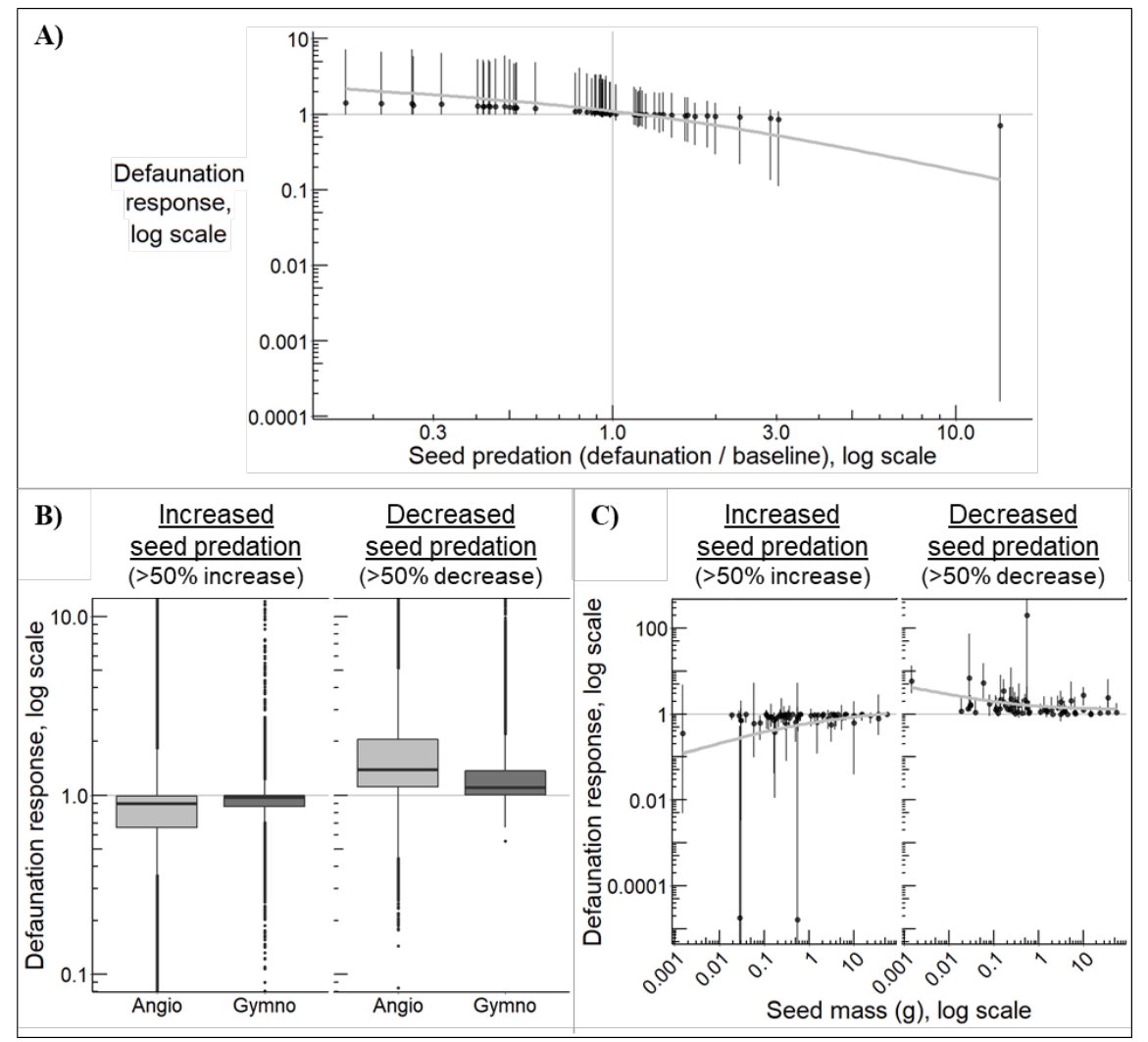

\section{Figure 3.}

Figure 1. Life cycle diagram for tree populations. The baseline and defaunation life cycles are identical except for the italicized terms. Under defaunation, the probability of dispersal $(d)$ changes for trees dispersed by large vertebrates but remains the same for other tree species. Seed survival ( $s_{s, u}$ and $\left.s_{s, d}\right)$ after defaunation is affected by changes in seed predation mortality. Seedling survival $\left(s_{s, u}\right.$ and $\left.s_{s, d}\right)$ after defaunation is affected by changes in trampling-induced mortality. Seedling survival in both baseline and defaunation scenarios is density dependent (see equation 1) based on the number of conspecific adults.

Figure 2. Relative importances of all variables (the percent of the variance in the data explained by each) included in our additive model in predicting the response of tree populations to defaunation. Dark gray bars show defaunation parameters, light gray bars show species traits.

Figure 3. Variables related to defaunation responses across all tree species and defaunation scenarios. 'Defaunation response' is the final number of adults in the defaunation scenario divided by the final number of adults in the baseline scenario. In panels $\mathrm{A}$ and $\mathrm{C}$, black circles represent the median defaunation response for each trait or parameter value; vertical black lines show the $95 \%$ confidence intervals. The trendlines show model fit based on all datapoints (not just median values) and include $99 \%$ confidence intervals that are too small to be seen. In panel B, the solid horizontial line shows the median, the box encompasses the $25^{\text {th }}$ to $75^{\text {th }}$ percentiles, and the vertical bars indicate the $95 \%$ confidence interval. There is clearly an inverse relationship between seed predation and defaunation response (panel A). Angiosperms and smallseeded species are more strongly influenced by defaunation - benefiting when seed predation is reduced and suffering when seed predation is increased; gymnosperms and large-seed species are less strongly affected (panels B and C). 